\title{
Control y evaluación de las políticas culturales en Chile ${ }^{1}$
}

\author{
Cristian Antoine $\left(^{*}\right)$
}

\section{RESUMEN}

Muchos países han asumido el imperativo de la evaluación de sus políticas públicas, incluyendo entre ellas a las llamadas "políticas culturales", las más jóvenes dentro del conjunto de intervenciones públicas en los asuntos de una nación.

No obstante, la interdependencia en la formulación de las políticas públicas en cultura con objetivos de interés general (public policies) y objetivos de carácter ideológico (public politics) de muchas de ellas, ha puesto en cuestión el si están suficientemente explicitados todos los propósitos que persiguen y su posibilidad de ser sometida a procesos de evaluación. La experiencia acumulada en otras naciones puede servir como referente a una discusión en ciernes sobre la realidad de las políticas culturales chilena. Pese a que Chile desarrolla una actividad evaluativa en torno a las políticas públicas que aplica, la evaluación de las políticas públicas en cultura sufre la poca experiencia que en tal sentido manifiesta la joven institucionalidad cultural del país.

Palabras clave:

Políticas culturales - evaluación - Consejo Nacional de la Cultura y las Artes.

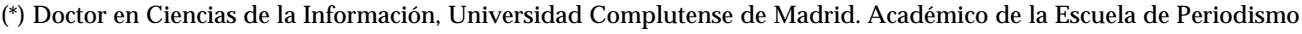
Universidad de Santiago de Chile.

Artículo recibido el 26 de enero de 2011. Aceptado por el Comité Editorial el 11 de abril de 2011

Correo electrónico: cristian.antoine@usach.cl

${ }^{1}$ Una versión ligeramente reducida de este trabajo fue presentada en el V Congreso Latinoamericano de Ciencia Política, organizado por la Asociación Latinoamericana de Ciencia Política (ALACIP), entre el 28 - 30 de julio de 2010, en Buenos Aires, Argentina. Este artículo está asociado al proyecto de investigación FONDECYT No 1085049, Análisis de las Políticas Culturales en Chile, Consumidores y Nuevas Audiencias. 


\begin{abstract}
Many countries have adopted the imperative of public policy evaluation, including among them the so-called "cultural policies", the newest in the group of public intervention in the affairs of a nation.

However, the interdependence in the formulation of public policies on culture with general interest objectives (public policies), and ideological objectives (public politics) many of them, have called into question if they are sufficiently explicit all the purposes they pursue and the possibility of being subjected to a process of evaluation. Experience gained in other countries can serve as a reference to an emerging discussion about the reality of the Chilean cultural policies. Although Chile develops an evaluative activity around the public policies that applies, evaluation of public policies on culture suffers from the limited experience in this respect manifests the country is young cultural institutions.
\end{abstract}

Key words:

Cultural policies - evaluation - National Council of Cultural and Arts.

\title{
I.
}

Es un antecedente conocido que los Estados asumen como un imperativo la evaluación de sus políticas públicas. Y lo hacen en todos los ámbitos de su accionar, incluyendo aquellos que por su naturaleza y conformación parecen ser más complejos a la hora de ser sometidos a examinaciones objetivas, como es el propio de la cultura y las artes.

Pero mientras algunas naciones promueven y ejecutan periódicas evaluaciones a sus políticas públicas en cultura, en otras -como en Chile por ejemplo- pese al conocimiento acumulado, parece que aún no se dan los primeros pasos. Estas diferencias no sólo ponen en evidencia los gap existentes en las culturas profesionales asociadas al dominio de conocimientos específicos en materias de conformación e implementación de políticas públicas de una materia particular, sino que, más trascendente aún, hablan de la necesidad de profundizar en este proceso. Toda vez que si las élites técnico-profesionales no lo promueven, poco más podrán hacer otros segmentos de la sociedad no llamados ni técnica ni académicamente a manejar este conocimiento. Aunque sean ellos sus depositarios finales, en su condición de ciudadanos, y contribuyentes.

Paralelo al desarrollo y conformación del cuadro académico de las políticas públicas, numerosos países fueron asumiendo la necesidad de introducir mecanismos que 
aseguraran al decisor (y a los órganos de control en cada país) contar con herramientas "objetivas" que permitieran saber cuándo un gobierno lo hace bien o no².

Los Estados Unidos de Norteamérica es un representante de ese primer grupo de naciones donde lo que producen quienes nos gobiernan, para lograr qué resultados, a través de qué medios -parafraseando la conocida alusión de Laswell- sobre las políticas públicas, ha dado lugar a un sistema formalmente institucionalizado de control de los productos del sistema político. También lo es Francia, país donde la institucionalidad de la cultura y las artes ocupa un lugar primordial en las preocupaciones públicas, y donde las primeras evaluaciones de las políticas culturales tuvieron lugar a fines de los años 80, habiendo generado desde entonces una amplia experiencia al respecto.

Mientras De Gregorio (2003) reconoce para el caso español en la ausencia de un fin claro, objetivo y cuantificable, la dificultad básica de la evaluación de las políticas culturales, no obstante el alto consenso en la finalidad de 'servir al interés general' que dicho proceso tiene. De modo que, con dificultades, España ha conformado una modalidad de evaluación de la acción pública en cultura que se ha convertido en una importante herramienta de gestión, un sistema de monitorización que valora ex post los programas que se han desarrollado, y determina lo que se ha hecho y cómo se ha hecho a partir de una planificación de los resultados ex ante.

Pulido (2001) afirma que para Argentina la evaluación de políticas públicas, con sus rigurosos métodos e instrumentos, tiene potencial impacto en el mejoramiento de la organización pública, tanto en sus modelos internos de gestión como en su intervención en el campo social a través de la producción de políticas, cobra un carácter crítico en un contexto latinoamericano caracterizado por una institucionalidad débil y por la desconfianza generalizada en el desempeño de la política.

La revolución liberal conservadora de los años ochenta acentuó esta atención a los fenómenos evaluativos, especialmente por el contexto de la crisis fiscal y las tensiones por la reducción/ampliación del tamaño del Estado. Como resultado de ese proceso, desde mediados de los noventa, se institucionalizó la necesidad de verificar la utilidad de los gastos públicos.

La evaluación de las políticas públicas llegó a ser una disciplina enseñada en las universidades y considerada en numerosas obras metodológicas de la especialidad. Tal vez, más por un reconocimiento pragmático que por una conclusión puramente

\footnotetext{
${ }^{2}$ Los mecanismos más habituales de evaluación de las políticas públicas en Chile suelen referirse a las siguientes perspectivas o dimensiones: Control de legalidad; Auditoría de gastos y eficiencia en uso de recursos; Cumplimiento de objetivos específicos; Impacto de cada política pública en materias como equidad, productividad y competitividad entre otros.

${ }^{3}$ Uso aquí la expresión en el sentido como bien lo desarrolla Squella (2005), de conjunto de los organismos de la administración del Estado (ministerios, servicios públicos, organismos técnicos, fondos concursables) que cumplen funciones en el ámbito de la cultura y que incluye además de las políticas culturales, a los planes y programas de trabajo gubernamental en el campo cultural, los organismos que ejecutan esos planes, el personal a cargo en esos organismos, los presupuestos públicos que financian a esos organismos, los instrumentos de asignación directa de recursos para el desarrollo cultural y, los estímulos de carácter estable que incentivan a la acción privada en el campo de la cultura.
} 
académica, los procesos de evaluación de las políticas públicas se han ido abriendo camino, con orígenes variados, en diversas latitudes.

La calidad de las decisiones públicas, requerirá entonces de una capacidad de evaluación y seguimiento de los impactos sociales y económicos de las leyes que se aprueban y de las políticas en las que se haya intervenido, directa o indirectamente (Ampuero, 2005).

La evaluación de las políticas públicas incluirá también al conjunto de decisiones que los Estados toman en el amplio campo del arte y la cultura, especialmente desde que el acceso y el disfrute de las mismas aparecieron consignadas como un derecho humano esencial en la Declaración Universal de los mismos, adoptada a fines de los cuarenta (Arroyo Yanez, 2006; Carmona Tinoco, 2002-2003).

Con una amplia gama de modelos teóricos y prácticas de campo, la evaluación de políticas culturales ha configurado un complejo cuadro de experiencias y trayectorias, siendo igualmente, muy difícil establecer un cuadro resumen de las mismas. No obstante, nos interesa en esta ocasión actualizar la cuestión a propósito del sentido que la evaluación de las políticas culturales alcanza en la actualidad.

En tanto "aetatis questionis", la siguiente comunicación propone una mirada descriptiva de la experiencia práctica y la reflexión teórica disponible a propósito de la evaluación de las políticas culturales en Chile. Asumimos adicionalmente que la evaluación de las políticas públicas en cultura debiera incluir la participación de los individuos y la sociedad civil interesada, para promover formas más inclusivas de democracia, pues ella introduce la necesidad de transparencia en las decisiones públicas.

En este cuadro el concepto de democracia está ligado también al de política cultural y, como una reseña de las prácticas evaluativas de las políticas culturales chilenas, la presente colaboración intentará mostrar que los actores concernidos no son plenamente considerados en estos procesos, los que suelen enfatizar los aspectos cuantitativos por sobre los cualitativos, las miradas institucionales por sobre las sociales $\mathrm{y}$, las referencias parciales a las globales.

\section{II.}

Evaluar los efectos de las intervenciones públicas contribuye a mejorar la racionalidad instrumental de la implementación de las políticas públicas, permitiendo conocer mejor la realidad sobre la que el decidor estatal interviene (comparación y objetivos), instando a la realización de análisis de la eficacia de la acción gubernamental (Rausell Koster, 2007).

Evaluar las políticas públicas es primero medir su eficacia mediante indicadores de desempeño y, en un segundo paso, determinar la relevancia de las mismas. La evaluación de las políticas públicas, que no debe confundirse con la evaluación y 
calificación de los funcionarios públicos, tiene dos dimensiones: una cuantitativa, que mide los efectos de la acción pública, comparando sus resultados con los objetivos y los medios empleados, incluyendo los indicadores de desempeño usado; y una cualitativa, que trata sobre la pertinencia de los objetivos.

Como cualquier otra política pública, una política cultural, consiste en una acción de gobierno basada en el análisis de la realidad y en un programa que pretende obtener unos objetivos de cambio y mejora (Ochoa Gautier, 2003). No obstante, habida cuenta la experiencia local en buena parte de las áreas de intervención del sector público, más allá de los campos del arte y la cultura, la práctica de la evaluación de las políticas culturales es aún una experiencia escasa y, por lo mismo, necesaria de reconocer allí donde nos parece se ha desarrollado con más suficiencia.

Estados Unidos cuenta con una larga tradición de estudios dedicados al análisis de las políticas culturales. Las ideas sobre la cultura han jugado un papel relevante en la historia del país, desde el principio de la organización de la república americana. Su experiencia en torno a la naturaleza de una ciudadanía que descansa en los valores de la libertad, está en la base misma de su concepción original de los derechos culturales.

No ausente de problemas, el "utopismo americano" en materias de políticas culturales, ha debido lidiar con la necesidad de hacerse cargo de una realidad multicultural, no necesariamente asumida en todos los planos de la vida del país.

La evaluación de las políticas públicas nació en Estados Unidos en 1932, seguido de la voluntad del gobierno por analizar los efectos de sus programas escolares (Bussiere, 2001) y, más tarde, otros ámbitos de intervención del gobierno.

Prueba al canto la entrega la evolución, desde 1967 al menos, del General Accounting Office (GAO), como unidad responsable en los Estados Unidos de evaluar a escala federal las políticas públicas. Ampuero (2005) ha subrayado su progreso desde una oficina menor en el establishment político norteamericano en los años veinte a ser en la actualidad la verdadera responsable de la evaluación de los efectos de las políticas públicas. Mientras que Jiménez Riuz (2003), la identifica como el resultado institucional más tangible de una serie de elementos favorables a la evaluación de políticas y programas públicos en EE.UU. Entre ellos, la adopción por parte de la administración americana de técnicas y métodos propios de la empresa privada (control de gestión, presupuesto por programas, etc.), los avances propios de las ciencias sociales y la concepción del Gobierno como prestador de servicios a los contribuyentes ('clientes'), desde una óptica similar a una contraprestación mercantil.

La variable de evaluación de las políticas públicas como un aspecto inseparable de la moderna gestión de gobierno ha sido descrita en numerosas ocasiones para el caso norteamericano (Gerrard, Ferroni, \& Mody, 2001; Jiménez Riuz, 2003; Palmer, 1999; van den Berg \& Boekmanstichting, 2008), contribuyendo a conformar un ambiente político, jurídico y legislativo muy favorable para su práctica. 
En efecto, era ya visible en Estados Unidos desde mediados de los setenta (Roller, 2005), la necesidad de responder a las demandas de información sobre el cumplimiento de las leyes que el Congreso norteamericano formulaba a distintos órganos del gobierno y el interés desde los altos cargos de la administración, interesados por conocer el verdadero impacto de las decisiones que se asumían.

La adopción de leyes asumidas con el carácter de sunset legislation ${ }^{4}$ por más de la mitad de los Estados de la Unión Americana, permitió contar con cuerpos legales que estaban conminados a ser evaluados exhaustivamente so riesgo de ser descontinuados. Se ha destacado que la filosofía que inspira a esta legislación tiende a bloquear la tendencia de los programas viejos a permanecer más allá del tiempo para alcanzar sus objetivos (Cardaval San Pedro, 2003), de modo que la previsión de la evaluación en los programas, es manifestación de la necesidad de una voluntad política que impulse y apoye la tarea de evaluación de programas en la administración y un alto grado de aceptación y formación de los técnicos como condición de éxito de la misma

La interdependencia en la formulación de las políticas públicas en cultura con objetivos de interés general (public policies) y objetivos de carácter ideológico (public politics) ha puesto en cuestión el si están suficientemente explicitados todos los propósitos que persiguen las políticas públicas sobre las artes y la cultura en un país. Pues allí donde hay objetivos explícitos también los puede haber implícitos, estos últimos están ligados a la gobernanza y al proceso mismo de toma de decisiones por parte de un gobierno.

Trabajos recientes publicados en ese país sobre políticas culturales (Bradford, 2000; Maya Cherbo, 2004; Mulcahy, 2001), han puesto de relieve la importancia de este campo de estudios. Los especialistas, artistas y responsables de formular las políticas culturales parecen haber reconocido la necesidad de moverse más allá de los debates sobre el cómo financiar desde el gobierno a las artes, hacia una serie de nuevas cuestiones en cuanto al papel de la cultura en la sociedad; la extensión de los principios del federalismo en el financiamiento del arte; los caminos innovadores que pueden ser encontrados para mejorar la estabilidad financiera de organizaciones de artes; la necesidad de un mejor entendimiento de los modelos internacionales de política cultural y la influencia sobre las políticas culturales de las transformaciones globales y los desafíos de ciberespacio, entre otras.

La misión de los analistas es averiguar quiénes son los stakeholders (Madden, 2005) de las políticas públicas en cultura. Lo que se busca es más transparencia. En Estados Unidos, por ejemplo las organizaciones artísticas tienen que rendir cuentas de sus

\footnotetext{
${ }^{4}$ No existe una traducción exacta de la expresión anglosajona "sunset legislation" [USA], a veces comprendida como "sunset act" o, en francés: "loi à durée déterminée" o "loi de temporarisation"; en alemán "Gesetz mit begrenzter Geltungsdauer"; en español suele aludirse a ellas como "ley de caducidad automática" o "ley con disposición de extinción automática". Se recomienda, por lo tanto, al menos en lo que respecta a documentos presupuestarios, la siguiente traducció n: cláusula de caducidad. En Boletines terminológicos y normativos, Boletín n 52, Unión Europea, disponible en http:/ /www.europarl. europa.eu/transl_es/plataforma/pagina/celter/bol52.htm
} 
actividades a su público y a quienes las financian; especialmente si están interesadas en recibir también fondos públicos ${ }^{5}$.

Mientras la evaluación de las políticas en el campo cultural reconoce también para la experiencia francesa, un caso ejemplar (Mollard, 2009; Negrier, 2003; Poirrier, 1999; Urfalino, 1997). En efecto, las primeras evaluaciones de las políticas culturales tuvieron lugar en la nación gala a fines de los años ochenta. Primero lo hicieron ciertos colectivos profesionales (Saint Pulgent, 1999), más tarde las ciudades interesadas en ponderar sus planes de desarrollo cultural (Saez, 2003). Una oportunidad interesante fue la conmemoración de los sesenta años de la creación del Ministerio de Cultura, en el 2009 (Caune, 2005; Mollard, 1999; Rout, 2005).

Entre los ochenta y los noventa el Estado francés aumentó al doble sus presupuestos para cultura, pero en paralelo se incrementó también la efervescencia de la discusión sobre la finalidad de la acción cultural pública.

El fenómeno evaluativo en Francia tuvo hacia los años setenta una fuerte revalorización, toda vez que apareció como una preocupación urgente en el aparato estatal, especialmente vinculados con la gestión de los presupuestos y la necesidad de fundar las decisiones de gasto sobre una previsión de su impacto socioeconómico (Bussiere, 2001). El procedimiento fue abandonado a mediado de los años ochenta, porque se influía sobre la elección del presupuesto.

En 1983, a raíz de un coloquio internacional sobre la evaluación de políticas públicas, organizado por el propio Ministerio de Hacienda, Francia tomó conciencia sobre su atraso en estas materias respecto de los países anglosajones y Europa del Norte. El desarrollo de las ciencias humanas contribuyó a una reflexión más certera sobre la calidad de las decisiones públicas. Se prefería el control administrativo ante una reflexión integral sobre la calidad de los servicios públicos.

Michel Deleau encabezó un grupo de estudio sobre la evaluación de las políticas públicas, avanzando en una definición que nos resulta de interés: evaluar una política pública es reconocer y medir (Deleau, 1986). El acento se puso sobre dos principios: primero, el hecho de volver transparente el método de evaluación y, segundo, la separación entre la concepción de la política y el evaluador. Mientras, en 1989 Michel Rocard confeccionó un informe sobre la evaluación en el contexto de la modernización del servicio público. Su rol en los procesos democráticos y el crecimiento eficaz del servicio público aparecieron en el informe, que también introdujo una nueva

\footnotetext{
${ }^{5}$ No obstante, la creación de un organismo nacional dedicado a la promoción de la cultura a mediados de los sesenta del siglo pasado, el National Endowment for the Arts (Fundación Nacional de las Artes), se ha traducido en el tiempo en un importante canal de recursos desde el gobierno federal a iniciativas locales que proporciona el mando y la dirección mientras recursos locales públicos y privados proveen la mayor parte de los fondos y servicios administrativos. "En EE.UU, los recursos estatales los administra el National Endowment for the Arts (Fundación Nacional de las Artes). Su ex presidente Frank Hodsoll explica que el presupuesto de esta agencia para este año es de US\$ 155 millones: “El $40 \%$ va para las agencias estatales de arte y el $60 \%$ es distribuido de manera directa. Opera con paneles de pares que evalúan las postulaciones. Así, al financiar un proyecto, se sigue el consejo de artistas, no de burócratas de Washington". El impacto de la entidad se midió al cumplir 35 años de vida, en 2000. Los teatros habían crecido de 56 a 340 . Las orquestas, de 980 a 1.800; las compañías de ópera, de 27 a 113, y las de danza se multiplicaron por 18". Cfr. (de La Sotta Donoso, 2009).
} 
definición de evaluación: "Evaluar una política pública es formar un juicio sobre su valor" (Bussiere, 2001).

Patrick Viveret agrega un entorno de "juicio de garantía": las garantías de independencia, competencia, rigurosidad, transparencia y pluralismo. Preconiza un desarrollo de la evaluación de las políticas públicas hacia una verdadera deontología de la acción gubernativa (Viveret, 1989). Propone también una interesante definición: "evaluar las políticas públicas consiste en medir los efectos que ellas engendran y buscar si los medios jurídicos, administrativos y financieros puestos en obra producen los efectos que se esperan".

Un coloquio realizado sobre el tema, en Grenoble en 1988, había puesto el acento en el modelo político de la acción cultural de las municipalidades y las ciudades (Des Politiques Culturelles, 1989). Las leyes de regionalización y las de transferencias de competencias, implementadas en Francia desde comienzos de los noventa, supusieron igualmente un estímulo al desarrollo de debates técnicos sobre las evaluaciones en el dominio cultural.

Las comunas han preferido compartir con el Estado sus obligaciones en materias culturales. Las Direction Regionale des Affaires Culturelles (DRAC) pueden evaluar sus acciones y sus políticas culturales. Los informes de evaluación no son siempre difundidos ni dados a conocer públicamente, algunos de estos informes han sido rotulados incluso como "confidenciales" (Bussiere, 2001).

Un ejemplo virtuoso es el de la ciudad de Rennes, que se ha preocupado por reflexionar sobre las políticas culturales que aplica. La ciudad ha pedido el apoyo a profesionales destacados que han colaborado con sus consideraciones (Gabillard, 2008). El evaluador resumió así su misión: "analizar la coherencia de la acción cultural regional así como el posicionamiento de la región en relación con otras intervenciones públicas". Para el estudio de la coherencia de la acción cultural regional el análisis abordó primero los objetivos comunes a todos los servicios de la institución regional, pero vistos desde el ángulo de las acciones culturales (desarrollo económico, empleo, managment del territorio), identificando los objetivos próximos al ámbito cultural.

El resultado de la evaluación de las políticas culturales de Rennes, según lo explica Bussiere (2001, p. 23) puso en evidencia la incoherencia del conjunto de la política cultural regional con la existencia de puntos débiles sobre la formación de personal y la coordinación entre los departamentos y servicios involucrados.

Y ello escapa con mucho a lo que podría aparecer como un exclusivo acento galo por la cultura y la ciudad, pues en el transcurso de estos últimos años, se han producido importantes cambios en la vida cultural municipal: expectativas gubernamentales más demandantes, nuevas necesidades de la población, presiones ejercidas sobre los bienes y servicios, restricciones presupuestarias, modas y tendencia. El diagnóstico puede ser extendido a muchas latitudes. Ocurre que los municipios sienten más que nunca la necesidad de captar mejor la realidad cultural y sus políticas. 
Las municipalidades francesas no son las únicas. En esta trayectoria, ya son varios los municipios canadienses (como el de Québec, por ejemplo), o españoles (Algeciras, Barcelona, Madrid), que podrían servir de referentes en estas materias al haber creado sus propios mecanismos de evaluación de las políticas culturales que aplican.

Por ejemplo, en el documento Itinerario21 Proceso de Evaluación Institucional de la Política Cultural de Algeciras: 1978-2003, ha permitido a ese ayuntamiento español ponderar su accionar en materias culturales en el contexto de una etapa superior de planificación en este ámbito (Cádiz, 2003). Del mismo tenor son las consideraciones contenidas en un documento catalán que avanza en la evaluación de sus museos (Museo de Arte Contemporáneo de Cataluña, el Museo Nacional de Arte de Cataluña, el Museo Nacional de la Ciencia y de la Técnica) y sus actuaciones estructurales en monumentos de gran dimensión física y simbólica (Montserrat, Poblet o Sant Pere de Rodes) (de Gregorio, 2008).

Lo cierto es que las políticas públicas reconocen cada vez más la necesidad de introducir criterios de evaluación y lo recogen en sus agendas culturales (Pascual i Ruiz, 2007).

Uno de los casos más completos de desarrollo de metodologías para la evaluación de las políticas públicas en cultura está radicado en España. Recientemente dada a conocer, la Guía para la evaluación de las políticas culturales locales (2009), es el resultado del trabajo compartido durante los últimos años por el amplio grupo de expertos designados por la Comisión de Cultura de la Federación Española de Municipios y Provincias de España en materia de sistemas de información cultural, evaluación e indicadores, con la colaboración del Ministerio de Cultura de España (Cultura, 2009).

La Guía contiene una amplia reflexión teórica sobre los temas más relevantes y acuciantes para los gobiernos locales en los que las políticas culturales tienen una mayor capacidad de progreso y transformación, y 316 indicadores, -cualitativos y cuantitativos-, que se proponen tanto para la política cultural desarrollada como para planificar con amplitud y ambición las políticas culturales que llevan a cabo. Esta publicación supone una experiencia pionera a nivel internacional, y la primera realización práctica de la Agenda 21 de la cultura en el ámbito de la construcción de herramientas para la planificación y la evaluación de las políticas culturales. La Agenda, alentada por Ciudades y Gobiernos Locales Unidos (CGLU) contiene los principios, compromisos y recomendaciones que se proponen a todos los gobiernos locales del mundo.

Pero hay experiencias más cercanas a nosotros. En Argentina, Rubens Bayardo ha documentado todo un conjunto de iniciativas de evaluación y seguimiento de la acción cultural, la creación de infraestructuras, el establecimiento de normativas y medios de financiamiento, y la planificación de programas y actividades orientadas desde el Estado (Bayardo, 2000).

En Colombia, un convenio para asesorar al Ministerio de Cultura en la consolidación del esquema de evaluación y seguimiento del Programa Salas Concertadas del 
Ministerio de Cultura a través del establecimiento de un sistema de indicadores de desempeño, ha propuesto una de las primeras y más completas formas de evaluación a las políticas y programas del Ministerio (de Colombia, 2007). Un aspecto que les preocupaba desde hace tiempo (Mena Lozano \& Herrera Campiño, 1994), pero que no habían logrado consolidar.

\section{III.}

¿Por qué el tema de la evaluación de políticas se ha ido convirtiendo con el paso del tiempo en un problema central de la acción de los gobiernos?

La primera de las tres razones que esbozan Raczynski \& Serrano (2002) es que el desarrollo y progreso social que se viven en el nuevo milenio no omiten ni aminoran la severidad de viejos y nuevos problemas sociales: pobreza, exclusión, desigualdad. La segunda, es que a pesar de las innovaciones institucionales y programáticas, las soluciones que se han puesto en marcha no arrojan los resultados esperados. La complejidad de nuestras sociedades, que presentan una muy desigual distribución de las oportunidades, y de los problemas que ello genera, exige miradas más acuciosas sobre las políticas y programas que se están implementando. La tercera se relaciona con la permanente escasez de recursos, cuestión que puede ser mejor enfrentada si la evaluación permite asegurar el uso eficiente de los recursos.

La cultura de la evaluación de las políticas públicas en Chile es un tema complejo, toda vez que no existe consenso entre los especialistas sobre la real vocación del sistema público chileno a examinarse con ojos críticos.

Algunos alegan que la autonomía de la formulación y evaluación de las políticas públicas en el país, ha tendido a ser exógena a la propia organización del Estado. Podría sostenerse, afirma (Jiménez Fernández, 2007). Aunque siempre hay excepciones, que el Estado chileno en sus funciones más propias ha renunciado a la capacidad de formular e incluso revisar las políticas que implementa:

\footnotetext{
“Durante el gobierno militar las políticas se generaron en el seno del propio Estado, si bien la influencia del grupo de los "Chicago Boy" y de los académicos de la Escuela de Economía de la Universidad Católica fue muy importante. A partir de comienzo de los ochenta, la oposición comienza a actuar a través de las ONG's y los centros de académicos independientes. En 1983, en Punta de Tralca, se reunieron alrededor de 500 profesionales cercanos a la DC para comenzar a buscar alternativas a las políticas públicas en aplicación. Del conjunto de organismos alternativos de ese periodo van a nacer los programas y equipos técnicos de la década siguiente. Por otra parte es interesante destacar que el mundo oficialista crea también sus propias entidades generadoras de propuestas y evaluaciones de políticas"(Jiménez Fernández, 2007).
}

Mientras que Olavarría Gambi (2008) ha destacado que las prácticas de evaluación de los actos del gobierno son de antigua data en el desarrollo institucional chileno, siendo al comienzo de nuestra historia institucional prácticas más bien formales, apegadas 
a criterios contables y legales -como eran las recomendaciones de la época-, pero a medida que fue avanzando el desarrollo conceptual, metodológico y disciplinario de las ciencias sociales se fueron sofisticando, dando paso a la noción de evaluación como una práctica diferenciada de las actividades de fiscalización y control ${ }^{6}$.

Los mecanismos de control existentes hoy en Chile apuntan al reconocimiento de la legalidad del accionar del Estado radicada en la Contraloría; la fiscalización de recursos públicos en la Cámara de Diputados; el seguimiento de la ley de presupuesto y su ejecución en la Comisión Mixta de Presupuesto; la auditoría interna (control de gestión informal) en algunos organismos y en la Dirección de Presupuesto (Auditoría Interna de Gobierno); la evaluación de la eficacia de algunos programas públicos (en la misma DIPRES); la existencia de algunas unidades de control de gestión de servicios públicos y la coordinación interministerial en el Ministerio Secretaría General de la Presidencia (Ferreiro, 2009).

Lo que muestra el desarrollo institucional chileno es, en perspectiva, una constante preocupación por el correcto uso de los recursos públicos. En los inicios la expresión correcto uso estuvo asociada al apego a la legalidad y, luego, el avance de las ciencias sociales y su instrumental ha ido adicionando prácticas de evaluación a lo largo del proceso de políticas públicas. Pareciera ser que la evaluación realizada hoy en Chile contempla una adecuada atención al control de gestión, pero se observa mayor debilidad en la evaluación de impacto.

Los primeros intentos por evaluar los logros de las políticas públicas datan de 1990, con el establecimiento de las "metas ministeriales", las que en 1995 se extendieron a los gobiernos regionales (Olavarría Gambi $(2008$, p. 3). Estas metas identificaban las prioridades de política pública que cada ministerio -y luego intendencias- debían cumplir cada año en el contexto del programa y objetivos del gobierno.

En los noventa, la generación de políticas públicas fue asumida en ocasiones por algunos think thank y universidades (como la Reforma Procesal Penal encargada a la Universidad Diego Portales), aunque el Estado de la naciente democracia chilena descansó su esfuerzo intelectual en lo que pudieron hacer sus propios equipos técnicos, emblemáticamente instalados en el "segundo piso" del Palacio de Gobierno.

Olavarría reseña que aunque el establecimiento de las metas ministeriales implicaron un avance, en términos de evaluación de políticas públicas, porque permitían desarrollar una visión del avance del gobierno en sus prioridades, evaluar los resultados e identificar las metas del año siguiente.

En enero y abril de 1996, la Mesa de la Cámara de Diputados y la Oficina de Informaciones organizaron un seminario sobre la eficacia de la ley, que resultó señero

\footnotetext{
${ }^{6}$ La primera práctica de revisión de los emprendimientos públicos, relata Olavarría Gambi (2008), data de 1541 cuando, luego de la Fundación de Santiago, el primer Cabildo designó a una comisión compuesta por cuatro oficiales con la misión de supervigilar el correcto uso de los fondos del reino.
} 
en estas materias (Ampuero 2005). Unos años más tarde, en 1998, se creó una Oficina en la Cámara dedicada el seguimiento presupuestario.

En trabajo conexos algunos autores (Andaur Troncoso, 2003; De la Maza, 2004; Lizardo Ovalle, 2001), han propuesto diversas metodología de análisis aplicadas a las políticas públicas desarrollados por el Gobierno en el período de la Transición Chilena (desde fines de los años ochenta hasta mediados de los noventa). En todos los casos, se trata de revisiones del recorrido histórico gubernamental sobre políticas de participación y gestión pública desarrolladas por el Gobierno, y patrocinadas por los grupos políticos dominantes, para luego a través del análisis buscar una explicación de los fenómenos específicos ocurridos durante los noventa, mostrando las particularidades de la situación chilena.

Esta práctica se habría luego burocratizado, durante el primer gobierno de la Concertación (P. Aylwin, 1990 - 1994), "adoleciendo de ambigüedad en la definición de metas y perdido significación en la toma de decisiones ministeriales" (Armijo, 2003).

La política de reforma y modernización impulsada por el gobierno siguiente, implicó un fuerte impulso a la expansión de las prácticas de evaluación en el gobierno (Ferreiro, 2009) ${ }^{7}$.

El presidente Eduardo Frei Ruiz - Tagle (1994 - 2000) se ha dicho (Cañas Kirby, 2003) que buscó hacer del Estado un agente de progreso, con un estilo de gestión orientada a resultados y con evaluaciones de logros alcanzados, tanto cuantitativos como cualitativos $^{8}$.

En el gobierno del presidente Ricardo Lagos (2000 - 2006), y como consecuencia de un acuerdo suscrito entre el Ministerio de Hacienda y el Congreso, a partir del 2002, la DIPRES desarrolla un tipo de evaluación denominada Evaluación Comprehensiva del Gasto?.

\footnotetext{
${ }^{7}$ Un papel relevante en este sentido lo desarrolla desde 1996 el Departamento de Evaluación de Programas Gubernamentales de la Dirección de Presupuestos de Chile (DIPRES) "organismo que asume la misión de mejorar la gestión a través del aprendizaje institucional de los encargados de implementar los programas públicos y del desarrollo de la evaluación y análisis de los resultados" y, por otro, "disponer de información que apoye la gestión, el análisis y toma de decisiones de asignación de recursos públicos", a la vez que reforzar la práctica de la rendición de cuentas públicas (De la Vega Rodríguez, 2006).

${ }^{8}$ A partir de 1998 este tipo de evaluación se hizo sólo con profesionales externos que debían estar inscritos en un registro de consultores del Estado. En el 2001 se incorporó la modalidad de Evaluación de Impacto, que es desarrollada por consultores externos seleccionados mediante licitación pública.

${ }^{9}$ Olavarría ha explicado que estas evaluaciones tiene por finalidad identificar la consistencia entre la misión institucional del ministerio, sus objetivos estratégicos, su estructura y distribución funcional, y los bienes y servicios que produce; su capacidad de implementar las políticas públicas encomendadas y los resultados que alcanza, en términos de eficacia, eficiencia, calidad y nivel de recursos comprometidos en sus logros (DIPRES 2003).
} 
Fruto de un acuerdo entre el gobierno y la oposición (2003) se consensuó una serie de medidas, algunas de ellas con referencias a la evaluación de programas y proyectos públicos $^{10}$.

Como una manera concreta de hacer frente a la necesidad de remozamiento del Estado, en el 2008 se creó, bajo el amparo del Ministerio del Interior, un Consorcio político-técnico pluralista, que pretende ayudar a concretar la idea de un aparato fiscal más eficiente y transparente, vinculando a la ciudadanía a fin de incidir en el diseño de políticas públicas en Chile ${ }^{11}$.

A partir de 2008 (Bravo, 2009) se constituyó una nueva línea de Evaluación de Programas que apunta a realizar evaluaciones rigurosas y científicas de las políticas públicas.

La Dirección de Presupuestos y el Centro de Microdatos de la Universidad de Chile -institución pionera en América Latina en esta materia- establecieron los criterios centrales: diseñar la evaluación al mismo tiempo que el programa o la política; considerar idealmente diseños experimentales (o bien cuasi-experimentales bien implementados). En cuanto a la evaluación, se estableció que deberá ser externa, y no puede estar vinculada con el ministerio o servicio que ejecuta el programa; de allí que la nueva línea utilice ejecutores externos; todo nuevo programa que implique una reforma estructural o un incremento mayor de recursos deberá tener una evaluación con estos estándares y, finalmente, se promoverá el desarrollo de pilotos que permitirá la evaluación de políticas antes de su implementación a gran escala ${ }^{12}$.

Una de las pocas experiencias de reflexión teórica sobre la evaluación de las políticas culturales es la que realizó el equipo dirigido por el sociólogo Pedro Güell en el marco de un asesoría del Programa de las Naciones Unidas para el Desarrollo (PNUD) al Consejo Nacional de la Cultura y las Artes de Chile (CNCA) referida a la generación de metodologías de evaluación adecuadas a los programas culturales y artísticos (Güell Villanueva, Frei \& Peters Núñez, 2008). No obstante, hasta donde sabemos esas metodologías propuestas no han sido implementadas o, si lo fueron, sus resultados no se han dado a conocer públicamente.

Los especialistas citados hacían ver que cada programa es una suma de componentes que se estructuran temporalmente, es decir, que tienen un diseño, una implementación, un resultado y un impacto que sucede en una lógica temporal. Existe, a su vez, un amplio conjunto de componentes de las políticas públicas que se repiten y que

\footnotetext{
${ }^{10}$ El denominado “Acuerdos Político - Legislativos para la Modernización del Estado, la Transparencia y la Promoción del Crecimiento", incluyó determinaciones sobre la necesidad de "crear un sistema nacional para la modernización del Estado, concentrando en su interior las funciones de evaluación ex - ante y ex - post de proyectos y programas públicos, así como la de auditoría interna"; y otra acerca de la consolidación de legislación sobre gestión financiera y difusión de programas y servicios públicos, que incluye una mención a la instauración de “... obligaciones de evaluación e información del presupuesto durante su ejecución ..."

${ }^{11}$ Más antecedentes en http:/ / www.reformadelestado.cl

12 “La nueva línea ha comenzado con la evaluación de cinco programas, entre los que se encuentra la reforma de pensiones, el subsidio al empleo juvenil, la Subvención Escolar Preferencial y el programa Chile Crece Contigo. Al cabo de dos o tres años podremos conocer si estas intervenciones están teniendo el efecto esperado o si deben ser modificadas en algún grado" (Bravo, 2008).
} 
pueden, por tanto, ser organizados en tipologías generales. Aunque si bien no existe un método único de evaluación para el conjunto de las políticas culturales y para su integralidad como programa, sí existen métodos y técnicas específicas para evaluar adecuadamente los distintos tipos de estos componentes (Ver Tabla $\mathrm{N}^{\mathrm{o}} 1$ ).

TABLA No 1

Métodos y Técnicas Específicas Para Evaluación de Políticas Públicas.

\begin{tabular}{|c|c|}
\hline Tipo de Evaluación & Mé to do \\
\hline Evaluación ex-ante & $\begin{array}{l}\text { Método de Análisis de Coherencia Lógica. } \\
\text { Método de Análisis de Consistencia General del Programa. } \\
\text { Método de Análisis Costo - Beneficio. } \\
\text { Método Análisis Costo - Impacto. } \\
\text { Método por Plan Piloto. } \\
\text { Método por Controles Experimentales. } \\
\text { Métodos por Diseños no Experimentales. }\end{array}$ \\
\hline $\begin{array}{l}\text { Evaluación Intermedia } \\
\text { o de seguimiento }\end{array}$ & $\begin{array}{l}\text { Método por seguimiento de beneficiarios. } \\
\text { Método de seguimiento presupuestario. } \\
\text { Método de seguimiento de los resultados inmediatos por productos. } \\
\text { Método de seguimiento de desempeño de ejecutores. }\end{array}$ \\
\hline $\begin{array}{l}\text { Evaluación ex - post o } \\
\text { de resultados }\end{array}$ & $\begin{array}{l}\text { Método de evaluación de resultados según diseño original. } \\
\text { Método de evaluación de la productividad de los integrantes del equipo ejecutor. } \\
\text { Método de evaluación de la población beneficiada directamente con el programa. }\end{array}$ \\
\hline $\begin{array}{l}\text { Evaluación de } \\
\text { eficiencia y/o } \\
\text { económica }\end{array}$ & $\begin{array}{l}\text { Método de cuantificación de los costos relevantes. } \\
\text { Método de análisis de eficiencia. } \\
\text { Método de análisis de economía. }\end{array}$ \\
\hline $\begin{array}{l}\text { Evaluación de impacto } \\
\text { social o longitudinal }\end{array}$ & $\begin{array}{l}\text { Método por diseño experimental. } \\
\text { Método por diseño cuasi experimental. } \\
\text { Método por diseño no experimental. }\end{array}$ \\
\hline
\end{tabular}

Fuente: Adaptado de Güell et. al. 2008.

En efecto, se aprecia en el cuadro adjunto cómo las Ciencias Sociales y la práctica de evaluación de políticas públicas a nivel nacional e internacional, han elaborado diversas 
técnicas de evaluación para los distintos tipos de componentes de los programas. En este sentido, evaluar un programa es, primero, reconocer la combinación específica de partes que lo componen; segundo, decidir la secuencia de técnicas que se adecua a esa combinación específica según la tabla indicada.

La evaluación ex ante, es un tipo de balance que se efectúa antes de poner en marcha una intervención, siendo su objetivo principal analizar su adecuación a las necesidades que la motivan y sus posibilidades de éxito. También se pronuncia sobre la coherencia interna de la lógica de planificación, la funcionalidad de los mecanismos de gestión y aplicación que se pretenden articular, sobre la idoneidad del sistema de seguimiento diseñado para recoger la información, y sobre los resultados e impactos previsibles del programa. Es, en definitiva, una evaluación prospectiva.

La evaluación intermedia (o de término medio), consiste en la valoración crítica de la información producida sobre el comportamiento de las actuaciones para determinar la pertinencia de los objetivos considerados, la calidad del sistema de gestión y seguimiento que se está aplicando, la forma en la que los objetivos perseguidos se están consiguiendo y, en definitiva, pronunciarse sobre la validez de la intervención en curso, explicando las diferencias respecto a lo esperado, y estimando anticipadamente su resultados finales ${ }^{13}$.

Mientras que la evaluación $e x$ - post tienen como objetivo emitir un juicio sobre el éxito o fracaso de la intervención desarrollada, el acierto de la estrategia diseñada, su grado de flexibilidad y capacidad de adaptación a una realidad siempre cambiante, su eficacia y eficiencia, la adecuación de los mecanismos de gestión y seguimiento aplicados, y los resultados e impactos logrados. Puesto que es necesario dejar transcurrir un tiempo desde la finalización de la intervención para que se manifiesten los impactos y efectos más indirectos, suele distinguirse entre evaluación final (realizada nada más terminar la intervención) y evaluación $e x$ - post (realizada en un momento posterior). Son evaluaciones retrospectivas.

La evaluación de eficiencia y/o económica consiste en una atención preferente a los elementos cuantitativos del desempeño del programa. Es probablemente la forma más usual de evaluación de políticas públicas que se realiza en Chile (DIPRES, 2005).

Por último, la evaluación de impacto social o longitudinal tiene por objetivo valorar los efectos más generales y menos inmediatos de la intervención sobre las necesidades de sus beneficiarios. Es un análisis complejo pues debe indagar en las relaciones de causalidad entre la intervención y los cambios habidos (efecto neto), deslindándolos de los efectos producidos por intervenciones distintas a la evaluada o por la propia evolución del contexto. Además, se debe indagar en la capacidad de replicar los

\footnotetext{
${ }^{13}$ No debe confundirse a la evaluación intermedia con el seguimiento. La diferencia radica en que el seguimiento permite informar, de manera aislada, de los elementos de la intervención (coherencia con otras intervenciones, ejecución y aplicación, grado de participación de la población objetivo, y evolución del contexto socioeconómico, entre otros), mientras que con la evaluación se relacionan todas las variables para emitir un juicio crítico sobre sus méritos y logros. Cfr. (Administración Pública, 2008)
} 
efectos positivos una vez retirado el programa, plan o política, es decir, la viabilidad y sostenibilidad de la intervención.

\section{IV.}

¿Ha tenido la novel experiencia chilena de formulación de políticas culturales la posibilidad de haber sido evaluada, en el contexto de lo que ya se sabe sobre evaluación de políticas públicas? ¿Existe un vínculo posible de establecer entre los recursos asignados estas dos décadas y los avances alcanzados, si es que hay avances?

La política cultural en los últimos años en Chile, es decir, desde el término de la administración militar y durante la sucesión de cuatro gobiernos democráticos de la Concertación de Partidos por la Democracia, encabezados por Patricio Aylwin, Eduardo Frei, Ricardo Lagos y Michelle Bachelet, no siempre ha sido formulada explícitamente, pero sí ha estado marcada por un esfuerzo que puede denominarse de democratización de la cultura (Antoine, 2004).

Se ha tratado por una parte, de eliminar los residuos más destacados de lo que fue la política cultural de la dictadura, si es que puede hablarse de ello, y, la otra, de responder a la naturaleza de un proceso de democratización política en el campo de la cultura.

Los logros y proyecciones de la acción cultural entre 1990 en adelante han sido destacados por varios autores (Bar, 2006; de Cea, 2006; Del Valle, Mayorga, \& Nitrihual, 2009; Squella, 2005) la mayoría de ellos reseñando con detalle los pormenores que permitieron dotar al país de un nuevo Consejo Nacional de las Artes y la Cultura (CNCA), pero con muy pocas o nulas alusiones a la evaluación de lo que el Estado ha intentado hacer en este campo ${ }^{14}$.

Existen algunas pocas tesis universitarias (Peralta Cabello, 2007; Vega Lavandeira \& Zepeda Farías, 2010) que han explorado el tema, constituyendo empero, una valiosa fuente de datos, aunque su aplicación es a escala muy reducida.

Es difícil saberlo, pues en elámbito cultural, pese a la existencia de una institucionalidad pública dedicada a estas materias desde mediados de la década, recién en diciembre del 2007 el Consejo Nacional de la Cultura y las Artes anunció que los fondos asignados por la vía de concursos, serían sometidos a un seguimiento más exhaustivo

\footnotetext{
${ }^{14}$ Algunas de las políticas culturales más importantes han correspondido a cuestiones institucionales y de financiamiento (Guerra Asenjo, 2003). En todo caso, es posible discernir, tres ejes dominantes: a) El primero de ellos es el establecimiento de instancias específicas para el desarrollo de la política cultural del Estado y de fuentes de recursos para la actividad cultural, es decir, el financiamiento de la cultura; b) El segundo es el apoyo a la actividad cultural y el fomento de la creación y las artes, las industrias culturales y c) El tercero tiene que ver con la democratización de la cultura en una doble dimensión: la de promover condiciones de libertad, expresadas en iniciativas como la eliminación de la censura cinematográfica y en la Ley de Prensa, y la de masificación del acceso a los bienes culturales, como la creación de centros culturales, descentralización de recursos como los del Fondart, eventos y encuentros como el Encuentro Nacional de las Artes, las Esquinas y Cabildos Culturales y las Fiestas de la Cultura, Cartografía Cultural, catastros y análisis y evaluaciones de creadores a nivel nacional, la Animación Cultural de Base con monitores-profesionales en diversas áreas, las iniciativas de Ciudadanía cultural, entre otras.
} 
que la tradicional verificación de ingresos versus gastos (Gayani Schele, 2007). No hay registros de que esas evaluaciones más exhaustivas se hayan hecho ${ }^{15}$.

Los Balances de Gestión Integral (BGI) del CNCA que están disponibles (2006, 2007, 2008 y 2009) conforman un insumo útil, más bien a los análisis sobre los niveles de cumplimiento de las metas institucionales en la ejecución de los presupuestos asignados (evaluación de eficiencia económica).

Tan sólo el correspondiente al año 2006 entrega algunas cualificaciones a criterios específicos asignados a algunos de los dieciséis objetivos estratégicos declarados para ese año. Los informes siguientes fueron hechos con una metodología diferente y no es posible deducir de ellos los efectos de las acciones implementadas sobre el campo cultural (evaluación de impacto).

Desde el año 2008 el CNCAha implementado en sus principales programas una "matriz de marco lógico", consistente con el impulso que ha tenido la evaluación ex - ante en otras reparticiones de gobierno, aunque la consultoría del PNUD a las estrategias de evaluación realizadas por el Consejo no encontró muestras de evaluaciones hechas en esta primera dimensión (Güell, 2008, p. 21).

Una atención preferente por parte de las autoridades del servicio ha estado puesta en auditar al Fondo Nacional de Desarrollo Cultural (Fondart) (DIPRES, 2010), aunque sus balances no han pasado de concluir en la necesidad de hace un seguimiento más estricto a los proyectos ${ }^{16}$.

Recién en la cuenta pública del Ministro Presidente del Consejo Nacional de la Cultura y las Artes de 2010, dado a conocer a pocos meses de instalado el gobierno, el titular de la cartera presentó algunas consideraciones sobre el cumplimiento de las metas más allá de la ejecución presupuestaria, insinuando una futura evaluación del impacto de las tareas que el Consejo realiza ${ }^{17}$.

El personero reconoció a la carencia de instrumentos de evaluación de las políticas que el CNCA implementa y la urgencia de implementarlos (Cruz - Coke, 2010).

\footnotetext{
${ }^{15}$ Pese al reclamo que algunas medidas en el ámbito cultural adoptadas por el Gobierno han despertado en la opinión pública interesada, exigiendo una mejor explicación, como en el episodio del "maletín literario" por ejemplo. La iniciativa fue anunciada por la Presidenta Bachelet en su cuenta anual del 21 de mayo de 2007. La idea era entregar libros a un conjunto de familias en atención a sus condiciones socioculturales. "El maletín literario permite ilustrar un principio básico que debiera formar parte de todas las iniciativas de gasto público, idealmente en la etapa de diseño de dichas iniciativas. Dicho principio es que estos programas deben ser diseñados de modo que se pueda evaluar en qué medida logran el objetivo propuesto" Cfr. (Engel, 2007).

${ }^{16}$ Creado en 1992 durante el gobierno de Patricio Aylwin, el Fondart nació como una manera de fomentar la creación y el desarrollo artístico y cultural del país a través del financiamiento de proyectos presentados en concurso público. El presupuesto se destina a respaldar distintas iniciativas, en cuyos análisis participan cerca de 500 especialistas.

${ }^{17}$ La Convención Nacional de Cultura es la principal instancia de encuentro de todos los órganos que constituyen la institucionalidad cultural chilena. En este espacio se analizan las orientaciones de la política cultural que impulsa el Consejo Nacional de la Cultura y las Artes. En la primera convención desarrollada en 2004, se inició un proceso de definiciones que sentaron las bases del documento "Chile quiere más Cultura. Definiciones de la política cultural 2005-2010". Desde ese entonces las convenciones han desarrollado temas como el desarrollo de la política en cada región del país, balances del proceso de instalación de la política, necesidad de espacios para la cultura y generación de más participación en ciudadana. Este año la Política Cultural 2005-2010 finaliza su período de vigencia de cinco años.
} 


\begin{abstract}
"Así como entendemos que la cultura es fundamental en el mejoramiento de la calidad de vida y el desarrollo integral de las personas, también es misión de una sociedad que se quiere justa, verla como una oportunidad de desarrollo. No podemos quedarnos de brazos cruzados cuando vemos que el incremento notorio que han tenido los mecanismos de fomento a la creación no se ha visto acompañado por un aumento sustantivo del consumo cultural, ni de las audiencias. En materia de audiencias, tenemos una realidad que reconocer: no estamos llegando a los más desprovistos de nuestra sociedad. Mientras en asistencia a eventos culturales el segmento social ABC1 promedia un 30,6\%, el segmento $\mathrm{E}$ lo hace con un desalentador $2,6 \%$. No podemos quedarnos sin actuar cuando vemos que el consumo cultural sólo aumenta en los segmentos más acomodados de la población. Mi desafío es muy ambicioso y el compromiso es disminuir esta desigual brecha que existe hoy".
\end{abstract}

En efecto, desde su creación el año 2003 a la fecha, el CNCA ha transitado por un período de instalación en el que se establecieron lineamientos para una política cultural nacional entre los años 2005 y 2010 y se aprobaron políticas regionales, además de otras para los sectores del Libro, Música, Audiovisual, Danza, Teatro, Artes Visuales y Fotografía. Si bien, su conformación supuso un importante avance político-institucional, existen aún desafíos para lograr su consolidación.

Aunque la inversión total en fondos concursables, por ejemplo, se ha incrementado respecto del 2005, fecha en que comenzó a operar la versión actual del sistema de fondos concursables, no existe una diferencia sustancial en los montos asignados a los programas ya existentes.

En el caso del Fondo de Fomento del Libro y la Lectura, la inversión es casi la misma del 2005 al 2008; sin embargo, la incorporación de dos nuevos fondos, Bicentenario e Infraestructura, implica un crecimiento sustancial de los números totales a partir del 2007. Por otro lado, no existe un sistema efectivo de medición, seguimiento y difusión de las iniciativas realizadas con los Fondos Concursables, es decir, un seguimiento posterior de los proyectos beneficiados con dichos fondos. No existe una evaluación de los proyectos tanto en su rentabilidad económica, como en su impacto social.

Con respecto a la evaluación de las rendiciones de los proyectos a nivel económico existe una demora sustancial en la entrega de certificados de rendición, dejando a los beneficiarios inadmisibles para postular a otros proyectos en el área.

La evaluación en la rendición de los proyectos sólo se realiza a nivel económico sin medir la calidad de los contenidos y la difusión de los proyectos, así como su impacto en la comunidad. En cierto sentido, es equivalente a decir que el Estado entrega recursos, pero no sabe qué se hace con ellos, más allá del gasto. Veamos algunos ejemplos ${ }^{18}$ :

\footnotetext{
${ }^{18}$ Me limito aquí por razones de espacio solamente a comentar ejemplos referidos a la línea de democratización de la cultura y generación de nuevas audiencias.
} 
El Programa Nacional de Fomento de la Creatividad en la Jornada Escolar Completa, Okupa en que participan 45 liceos subvencionados de las regiones de Valparaíso, Maule, Bío-Bío, Los Ríos, Los Lagos y Metropolitana, permite a cada liceo participante de $7^{\circ}, 8^{\circ}$ de Enseñanza Básica y $1^{\circ}, 2^{\circ}$ y $3^{\circ}$ de Enseñanza Media, tener la posibilidad de ofertar a sus estudiantes cinco talleres en las especialidades artísticas de música, artes visuales, audiovisuales, danzarias, teatrales, literatura y cultura tradicional, entre otras alternativas.

El fin o propósito de este programa es promover que las/los jóvenes adquieran una mirada más amplia y cercana respecto del hecho artístico, primero más inclusiva de la propia cultura de cada estudiante y luego, del entorno cultural donde el establecimiento educacional esté inserto.

Okupa es uno de los pocos programas de intervención cultural directa del Estado que ha sido sometido a evaluaciones exhaustivas en el pasado (De la cultura y las Artes, 2007c), tanto en el plano de los resultados como en el de los procesos ${ }^{19}$.

Por su parte, el Programa de Escuelas de Rock, es un Programa Nacional del Departamento de Ciudadanía y Cultura del Consejo Nacional de la Cultura y las Artes, destinado a estimular y difundir el desarrollo de la música popular chilena, promoviendo el acceso y la participación cultural de los jóvenes, fomentando el desarrollo de sus capacidades de expresión musical. El programa Escuelas de Rock busca fidelizar audiencias y públicos a través de procesos de formación artística, gestión y difusión en rock y música popular chilena.

No obstante, su declaración a la formación de públicos y la participación cultural, el Programa de Escuelas de Rock no ha sido sometido a evaluaciones en tal sentido, así que no podemos saber si sus expectativas se han logrado.

Creando Chile en mi Barrio, tiene como propósito contribuir al mejoramiento de las condiciones de desarrollo artístico y cultural de las personas que viven en poblaciones económicamente vulnerables y geográficamente distantes. A través de un enfoque gubernamental participativo este programa de animación sociocultural desde el Estado estimula los procesos de co-producción de estrategias y actividades que representen las demandas, motivaciones y experiencias de la ciudadanía promoviendo, al mismo tiempo, el fortalecimiento de su asociatividad. Desde su creación en el 2006, el proyecto no ha sido sometido a evaluaciones más allá de su ejecución presupuestaria.

\footnotetext{
19 “En el ámbito del desarrollo de capacidades creativas de los alumnos en cuanto al aprendizaje y perfeccionamiento de saberes y conocimientos, se concluye que la mayoría de los alumnos participantes pudo, por intermedio de los talleres, adquirir nuevos conocimientos en el ámbito artístico cultural y/o perfeccionar los que ya tenían, si bien hay que considerar que en cada grupo se observan niveles diferenciales de aprendizaje y; en cuanto a las prácticas de consumo cultural que realizan los jóvenes, se concluye que tras los talleres hay un aumento en el consumo de bienes culturales, en la lectura de periódicos, revistas y cómics, en la visita a exposiciones y en la asistencia al teatro y la Danza". Cfr Consejo Nacional de la Cultura y las Artes, Programa Okupa.
} 
Al examinar la información disponible sobre evaluación de políticas y programas culturales en Chile se observa, en consecuencia, que las preocupaciones se han centrado en cuestiones teóricas y metodológicas, institucionales y operativas, mientras en otras naciones se han preferido las evaluaciones de impacto, aún a escala local, municipal y/o regional.

También se advierte que existe una tendencia a desarrollar modelos de evaluación dedicados al proceso, sin mayores consideraciones sobre el resultado, impacto y satisfacción de los usuarios por la implementación de las mismas.

En la mayoría de los casos, los actores que participan de las decisiones sobre evaluación son los directivos y autoridades, así como los operadores de las intervenciones y los profesionales de las ciencias sociales que realizan labores de evaluación ${ }^{20}$.

Quienes se ven concernidos por la aplicación de las políticas culturales -en primer lugar, los ciudadanos que se ven afectados por la implementación de las decisiones de los gobiernos y sus programas y, luego los artistas y las organizaciones culturales encargadas de su gestión- no parecen estar siendo plenamente considerados en este proceso.

\section{Fuentes y Referencias}

Administración Pública, I. d. Glosario de Términos relacionados con el seguimiento y evaluación de las Políticas Públicas (Glosario). Pamplona, Navarra, España, 2008, Gobierno de Navarra.

Ampuero, H. La Evaluación de la Ley (o Cómo las salchichas y las leyes deben cumplir su cometido)2005, 489. Retrieved from www.asuntospublicos.org

Andaur Troncoso, R. T. Implementación del fondo de proyectos de mejoramiento educativo del Ministerio de Educación, 1992-1997: un caso de gestión innovadora de políticas públicas en educación, 2003, Santiago.

Antoine, C. "La Nueva Institucionalidad cultural de Chile". [paper]. Revista Ius Publicum(12), 2004, 89.103.

Armijo, M. “La Evaluación de la Gestión Pública en Chile”. En N. Cunill Grau \& S. Ospina Bozzi (Eds.), Evaluación de Resultados para una Gestión Pública Moderna y Democrática. Experiencias Latinoamericanas", 2003, Caracas, CLAC.

\footnotetext{
${ }^{20}$ Prueba de ello son la recientemente creada Unidad de Estudios en el Ministerio de Educación y los planes similares que tiene el CNCA para reforzar una similar que, con vicisitudes, mantiene funcionando desde el 2004 en sus oficinas centrales en Valparaíso.
} 
Arroyo Yanez, L. M. (2006). “Los derechos culturales como derechos en desarrollo: una aproximación". Nuevas Políticas Públicas; Anuario multidisciplinar para la modernización de las administraciones públicas. Ejemplar dedicado a los derechos sociales(2), pp. 262 - 283.

Bar, N. ¿Qué hay detrás del Nuevo Consejo Nacional de la Cultura y las Artes en Chile? Fundamentos para la Nueva Institucionalidad. Universidad de Warwick, Inglaterra, Warwick, 2006.

Bayardo, R. (2000). Políticas Culturales en la Argentina, 2000, p. 6.

Bradford, G. The Politics of Culture: Policy Perspectives for Individuals, Institutions, and Communities, 2006.

Bravo, D. Evaluando los programas y las políticas públicas. El Mercurio. Retrieved from http:/ /diario.elmercurio.com, 27 de agosto de 2009.

Bussiere, A. L'Evaluation des politiques culturelles, entre rationalisation et démocratie. [Memoria]. (Memoire DEES “Developpement culturel et direction de proyect"), 2001, p. 63.

Cádiz, U. d. Itinerario21 Proceso de Evaluación Institucional de la Política Cultural de Algeciras: 1978-2003. Cadiz, Universidad de Cádiz- Fundación Municipal de Cultura, 2003.

Cañas Kirby, E. "Modernización de la Gestión Pública del Estado de Chile". En Ó. Muñoz \& C. Stefoni (Eds.), El Período del Presidente Frei Ruiz - Tagle. Santiago, Editorial Universitaria, 2003.

Cardaval San Pedro, M. "Consideraciones en torno al gasto público". [paper]. Revista Galega de Economía. Universidad de Santiago de Compostela, España, 12(001), 2003, p. 9.

Carmona Tinoco, J. U. "El derecho humano a la cultura y su protección internacional". [paper]. Revista Derecho y Cultura, Invierno 2002-2003.

Caune, J. "La politique culturelle initiée par Malraux". [Paper]. EspacesTemps.net (Faculte de l'environnement naturel, architectural et construit (ENAC), Ecole polytechnique Federale de Lausanne), 2005.

Cruz - Coke, L. Cuenta Pública VII Convención Nacional de la Cultura. Valparaíso, 2010.

Cultura, C. d. Guía de Evaluación para las Políticas Culturales Locales. Madrid, Federación Española de Municipios y Provincias de España, 2009. 
De Cea, M. “Hacia una nueva institucionalización de la experticia cultural en Chile. El rol del Consejo Nacional de la Cultura y las Artes". In E. U. Bolivariana (Ed.), Qué tipo de nexos para qué tipo de políticas. Estudio comparado en diversos ámbitos de intervención pública en Chile. Santiago, Chile, 2006.

De Colombia, U. N. Esquema de evaluación y seguimiento del Programa Salas Concertadas del Ministerio de Cultura (Report). Bogotá, Facultad de Ciencias Económicas, Centro de Investigaciones para el Desarrollo / Ministerio de Cultura, 2007.

De Gregorio, A. La dotación de equipamientos culturales públicos en Cataluña: concertación, planificación y responsabilización. Paper presentado a las Jornadas sobre evaluación externa de proyectos culturales. Retrieved from http://www. ub.edu/cultural, 2008.

De la Maza, G. “Políticas Públicas y Sociedad Civil en Chile: El Caso de las Políticas Sociales (1990-2004)". [Paper]. Política, 2004, pp. 43-44.

De la Sotta Donoso, R. El cara a cara de dos modelos culturales. El Mercurio. Retrieved from http:/ / diario.elmercurio.com, 21 de junio de 2009.

De la Vega Rodríguez, L. F. (2006). Evaluaciones de programas gubernamentales de DIPRES. Magíster, FLACSO, Santiago de Chile, 2006.

Del Valle, C., Mayorga, A., \& Nitrihual, L. Políticas culturales en Chile: popularización, invisibilización y politización. [Paper]. Legete. Estudios de Comunicación y Sociedad. Escuela de Periodismo, Universidad Católica de la Santísima Concepción(12), 2009 , pp. $25-42$.

Deleau, M. Evaluer les politiques publiques : mthodes, dontologie, organisation: rapport du groupe de travail "Mthodes d'valuation des politiques publiques". Paris, La Documentation Franaise, 1986.

Des Politiques Culturelles, O. Coopération des collectivités publiques et action culturelle : compte-rendu du colloque de Grenoble (1-2 décembre 1988). Paris. Documentation française, 1989.

DIPRES. Metodología Evaluación de Impacto. Santiago, Ministerio de Hacienda, Dirección de Presupuesto, División de Control de Gestión, 2005.

DIPRES. Informe de Cumplimiento de compromisos al 31 -12- 2009 del Fondo Nacional de la Cultura y las Artes (FONDART) (Reporte). Santiago, Dirección de Presupuesto, 2010.

Engel, E. “Evaluando el impacto del maletín literario". La Tercera, 2007. 
Ferreiro, A. "Agencia de Calidad de las Políticas Públicas". En F. Silva (Ed.), Consorcio para la reforma del Estado. Santiago, Chile, 2009, p. 14.

Gabillard, M. La politique culturelle à Renne, 1977 - 2008: Memoires et réflexions. Renee, Francia, Apogée, 2008.

Gayani Schele, G. Ministra de Cultura anuncia cambios en fondos concursables, 2008, El Mercurio, 13 de diciembre de 2007.

Gerrard, C. D., Ferroni, M. A., \& Mody, A. Global public policies and programs : implications for financing and evaluation: proceedings from a World Bank workshop. Washington, D.C., World Bank, 2001.

Güell Villanueva, P., Frei, R., \& Peters Núñez, T. Propuesta de Metodologías de Evaluación de Programas Culturales y Artísticos (Report). Santiago, PNUD Chile, 2008.

Jiménez Fernández, G. ¿Cómo se hacen las Políticas Públicas en Chile?. Santiago de Chile Centro de Estudios Sociales Avance, 2007, p. 2.

Jiménez Riuz, P. “La Evaluación de políticas públicas en los Estados Unidos y el papel de la General Accounting Office". [Paper]. Auditoría y Gestión de los fondos públicos(31), 2003, p. 31.

Lizardo Ovalle, G. V. Las políticas públicas implementadas para la educación básica en República Dominicana durante el período presidencial 1996-1999. Universidad de Santiago de Chile, USACH, Santiago, 2001.

Madden, C. "Indicators for arts and cultural policy: A Global perspective". Cultural Trends, 14(3), 2005, pp. 217 - 247.

Maya Cherbo, J. The Public Life of the Arts in America, Rutgers University Press, 2004.

Mena Lozano, U., \& Herrera Campiño, A. R. Políticas Culturales en Colombia. Discursos estatales y Prácticas Institucionales. Bogotá, M\&H Editoras, 1994.

Mollard, C. Le Cinquieme Pouvoir - La Culture Et L'etat De Malraux À Lang. París, Armand Colin, 1999.

Mollard, C. L'Ingenierie culturelle et l'evaluation des politiques culturelles en France. Paris, Presses universitaires de France, 2009.

Mulcahy, K. The State Arts Agency: An Overview of Cultural Federalism in the United States, The Cultural Policy Center, University of Chicago, 2001, p. 24. 
Negrier, E. Las Políticas culturales en Francia y España: una aproximación nacional y local comparada. Barcelona, Institut de Ciencies Politiques i Socials (ICPS), 2003, p. 39

Ochoa Gautier, A. M. Entre los Deseos y los Derechos. Un Ensayo Crítico sobre las Políticas Culturales. Bogotá, Colombia, Instituto Colombiano de Antropología e Historia, 2003.

Palmer, J. Book Review: Public Policy and Program Evaluation (Vedung, Evert) American Journal of Evaluation(20), 1999, p. 5.

Pascual i Ruiz, J. “Guía para la participación ciudadana en el desarrollo de políticas culturales locales para ciudades europeas". En S. Dragojevic (Ed.). Barcelona, España, Fundaciòn INTERARTS / Asociación ECUMEST / Fundación Europea de la Cultura, 2007, p. 70.

Peralta Cabello, P. . Procesos de Supervisión, seguimiento y evaluación a proyectos beneficiados mediante concurso público. El caso del Fondo Nacional de Fomento del Libro y la Lectura. Universidad de Chile, Santiago, 2007.

Poirrier, P. "Bibliographie de l'Histoire des politiques culturelles. France XIX - XX siecles", Travaux et Documents $n$ 9. París, Collection du Comité d'histoire du Ministère de la Culture et de la Communication, 1999, p. 221.

Pulido, N. Evaluación Transversal de políticas públicas. Gestión de la participación ciudadana. Paper presentado al Seminario Internacional "La reinvindicación de la Política y la Ciudadanía". Retrieved from http:/ / www.auditoriaciudadana. gov.ar, 2001.

Raczynski, D., \& Serrano, C. La función de evaluación de políticas sociales en Chile. Retrieved from http:/ / www.asesoriasparaeldesarrollo.cl, 2002.

Rausell Koster, P. Apuntes y Herramientas para el Análisis de la Realidad Cultural Local. En J. Martinez Tormo (Ed.). Valencia, Unidad de Investigación en Economía Aplicada a la Cultura, 2007, p. 25.

Roller, E. Performance of democracies:political institutions and public policies. Oxfored, UK ; New York, Oxford University Press, Inc, 2005.

Rout, C. La diversité au sein des politiques du ministere de la culture: rappel historique. Culture \& Recherche, 60, diciembre 2005.

Saez, G. Les politiques culturelles des villes. Du triomphe du public á son effacement. In O. Donnat \& P. Tolila (Eds.), Le (s) Public (s) de la culture. Paris, Presses de Sciences Politiques, 2003, pp. 197-226. 
Saint Pulgent, M. d. Le Gouvernement de la Culture. Paris, Gallimard, 1999.

Squella, A. El Jinete Bajo la lluvia. La cultura en el gobierno de Ricardo Lagos. Santiago, Chile, Aguilar, 2005.

Urfalino, P. Quelles missions pour le ministere de la culture? Esprit, janv 1997, p. 24.

Van den Berg, H. O., \& Boekmanstichting, E. State on stage : the impact of public policies on the performing arts in Europe. Amsterdam, Boekmanstudies, 2008.

Vega Lavandeira, M., \& Zepeda Farías, G. Análisis del Programa Nacional de Centros Culturales del Consejo Nacional de la Cultura y las artes: Infraestructura y Audiencias. Magíster, Universidad de Chile, Santiago, 2010.

Viveret, P. L'evaluation des politiques et des actions publiques. París, La Documentation Française, 1989. 